\title{
EEG CHANGES DURING READING IN ADHD CHILDREN
}

The relation between electroencephalographic (EEG) findings, behavioral, and continuous performance test indicators were examined in 23 boys, aged 9-11 years, with attention deficit hyperactivity disorder (ADHD) and 23 matched controls in a study at the State University of New York, Cortland, NY, and University of Tennessee, Knoxville, TN. Compared to controls, the EEG results showed increased alpha in posterior regions during baseline for subjects with ADHD. Boys with ADHD showed decreased alpha in the left frontal regions during reading, and increased alpha activity at T5 during a coding task. The ADHD group had poorer performance on the TOVA test than normal controls. (Swartwood JN, Swartwood MO, Lubar JF, Timmermann DL. EEG differences in ADHDcombined type during baseline and cognitive tasks. Pediatr Neurol March 2003;28:199204). (Respond: Dr Swartwood, SUNY Cortland, Psychology Department, Cortland, NY 13045).

COMMENT. The electroencephalogram in children with reading disabilities has been studied for more than 30 years. Hughes JR and Park GE (Electroencephalogr Clin Neurophysiol 1969;26:119) found abnormal EEGs in 36\% of 157 dyslexic children of normal intelligence. Abnormalities were in 4 groups: 1) those with positive spikes had dyslexia but the highest IQs; 2) those with occipital slowing included the poorest readers; 3) frontal temporal EEG abnormalities were associated with reading disabilities and ocular deficiencies; and 4) epileptiform EEGs with evidence of organic brain damage. An EEG experiment aimed toward identifying dyslexic children (Sklar B et al. Nature 1972;240:414-416) found a higher theta-band activity as the most recurring difference from EEGs of normal children.

The EEG abnormalities in the children with ADHD were task and bandpass specific. Significantly more alpha was identified at T5, T6, and O2 during the eyes open baseline condition, when compared to non-ADHD controls. Participants with ADHD fail to suppress alpha activity with eyes open, a finding consistent with the inability to process visual stimuli efficiently. Alpha persistence in the left parietal region during a coding task is indicative of parietal underactivation in ADHD. The finding of increased left frontal alpha activity during reading in both normal controls and ADHD children treated with MPH (Swartwood et al 1998) may indicate inactivation of that region.

Left frontal cortex activation in reading epilepsy. Spike-related fMRI activity was found in the left precentral gyrus, and spread to both central sulci and globus pallidi, in a 15-year-old girl with reading epilepsy. (Archer JS et al. Neurology 2003;60:415-421). An associated localized structural abnormality caused the spikes to spread from left middle frontal working memory areas into adjacent motor cortex.

\section{COGNITIVE EFFECTS OF TOPIRAMATE AND VALPROATE}

Cognitive and behavioral effects of topiramate (TPM) and valproate (VPA) as adjunctive therapy with carbamazepine (CBZ) were compared in 62 adults (16 to 55 years old) with refractory partial seizures, in a randomized, double-blind trial at the Medical College of Georgia, Augusta. After a 4-week baseline, the study drug was titrated over 8 